\title{
Production of resistant starch type III from native sago starch as a potential prebiotic
}

\begin{abstract}
Resistant starch (RS) is the sum of starch that escape upper gastrointestinal digestion and can be fermented by colonic microorganisms to produce beneficial metabolites such as short chain fatty acids (SCFA). Resistant starch can be a potential prebiotic, a non-digestible food ingredient that selectively stimulate the growth and/or activity of gut microorganisms, thus promoting the host health. The main focus of this study is to produce resistant starch type III from native sago starch. The resistant starch was produced via retrogradation process and with the aid of pullulanase enzyme. The quantification of total resistant starch content was determined based on the method approved by AOAC 2002.02 and AACC 38-40.01. From this study, it showed that there are no significant different between the resistant starch content of native and modified sago starches (RS type III).
\end{abstract}

Keyword: Resistant starch; Prebiotic; Retrogradation; Sago starch 